\title{
The Fuzzy Processing of Metaphors
}

\author{
Charles Tijus $^{(\bowtie)}$ (D) \\ Cognitions Humaine et Artificielle, Université Paris 8, Saint Denis, \\ 93526 Saint Denis, France \\ tijus@lutin-userlab.fr
}

\begin{abstract}
The question under investigation about verbal metaphor is what kind of thinking and reasoning can help catching the metaphorical target category (e.g., octopus meaning citrus press, child meaning son or daughter, pizzas meaning people) from a source category (children, pizzas or octopuses); knowing that this kind of "logically false" way of talking/understanding and of reasoning appears to be the most prominent kind of human of thinking. We are reviewing some of the prominent work of Bernadette Bouchon-Meunier with her team to evaluate how much the fuzzy logic computation of metaphorical reasoning and schemes can be used to model the human computation of metaphors.
\end{abstract}

Keywords: Thinking $\cdot$ Reasoning $\cdot$ Similarity $\cdot$ Analogy $\cdot$ Metaphor

\section{Introducing Metaphor as One of Main Ways of Thinking and Reasoning}

Let's imagine three situations

1) A grandmother and her grandson

- The grandson: Granny, is Dad your child?

- Grandmother: Yes, he is my child

- The grandson: Yes but yet, dad is no longer a child!

2) A waiter of a restaurant and a group of 3 friends who have lunch together in this restaurant

- The first client says to the waiter: I'm going to have a pizza!

- The second client: me too!

- The third client: I too will have a pizza.

- (after the meal, the customers pay and leave)

- The waiter shouting: hey you! The 3 pizzas, you're forgetting your change!

3) Philippe Starck, who is a designer, invented his famous citrus press that has an octopus shape, named Juicy Salif, by drawing on the paper tablecloth of an Italian restaurant, while tasting octopus seasoned with lemon. People that see Juicy Salif are usually saying "oh this is an octopus!". 
Homonyms are words that are spelled the same and sound the same way but have different meanings. In these three situations, either child, pizza or octopus has two different meanings although they are not homonyms:

- one meaning relates to a known category of things (children, pizzas or octopuses) and is properly used (child used by the grandson as being a boy or girl from the time of birth until he or she is an adult; pizza as a large circle of flat bread baked with cheese, tomatoes, and so on or octopus as a sea creature with a soft, oval body and tentacles), while

- the other meaning relates to a different category (child used by the grandmother as being a son or daughter of any age, pizzas to denote customers, octopus to represent a citrus press).

Thus, the question under investigation is what kind of thinking and reasoning can help catching the metaphorical (e.g., octopus meaning citrus press), pragmatic (e.g., child meaning son or daughter) or metaphorical target category (e.g., pizzas meaning people) from a source category (children, pizzas or octopuses); knowing that this kind of "logically false" way of talking/understanding and of reasoning appears to be the most prominent kind of human thinking [1]. From now, we label "metaphor", from the Latin "metaphora", itself from the Greek " $\mu \varepsilon \tau \alpha \rho о \rho \alpha$ ", a figure of style based on attributive categorization [2]. It designates a target thing (e.g., some specific people being called as "pizzas") with the name of another category (pizza) as a source for attributing to the target thing some of the features of the source category (have a pizza for lunch). Thus, instead of saying "Hey you people that were eating pizzas", they can be named and recognized as the meanings of "pizzas" in a sentence such as "hey you, the pizzas, you have forgotten your money".

\section{The Logic Behind Metaphor Thinking}

Associations, - such as Juliet is my sun (she brings me joy and shine) in the Romeo's diary of Shakespeare's Romeo and Juliet-, are in fact based on some fuzzy resemblance, on analogy or metaphor (upon, according to/so to speak) [1]. Analogy is usually based on similarity of things and on a computation based on "partial agreement, likeness or proportion between things", i.e. for transfer of learning. Although such metaphorically-based reasoning is providing sentences that do not have an evidential support and are logically "false", this kind of reasoning appears to be the most prominent kind of human of thinking [1].

Although Metaphors seem to be produced through metaphorical reasoning based on comparison (e.g., "Peter is a dog"): Peter, who is a troubadour, sings to beg for a little love. Droopy, who is a dog, barks to beg for food. Peter's voice sounds like a barking: a proposition such as "Jean is barking" is an analogy, while "Peter is a dog" is a metaphorical proposition [2] based on attributive categorization.

Unlike deduction which provides "true" conclusions, the human inferences which produce a metaphor (major premise: a person had a meal, minor premise: the meal was pizza, conclusion: this person is a pizza) concludes with a false expression (not literal; not legally comparable). In such way of thinking, the things that are under cognitive 
investigation (e.g. the people forgetting their change in a restaurant) that are used to produce metaphors are of inter-domain constraints (people are not food). Although they are of different categories, generally of exclusive domains, inference is made by starting from an example of a given category and ends with another different category.

First, note the process is done from target-to-source to produce a metaphor (to produce Juliette is my sun: from Juliette, Shakespeare had to find a source of resemblance) whereas to understand the resemblance of the source, the cognitive inquiry of the listener is a process from source-to-target (to understand that Juliette is my sun: from the sun, the listener must find the resemblance that is to be to attributed to Juliette) [3].

Note also that if the metaphor is based on implication ( $\mathrm{x}$ is a kind of $\mathrm{y}$ ), the source (that serve as vehicle to transmit meanings) and the target (that is the topic) cannot swap: "Peter is a dog", the reverse is not true: dogs are not like John [2]. This is somewhat an asymmetric similarity measure on sets, not to compare a variant to a prototype [4], but a prototype to many variants.

In fact, there is a scientific and technological problem that is very difficult to solve: the what and the how of thought processes that are capable of producing and understanding metaphors. What type of calculation linking a target category to a source category belonging to another domain could be able to support reasonings based on imperfection, imprecision and approximation, graduality, imprecision, blurring, uncertainty and plausibility.

It seems to us that significant advances in the metaphor resolution stem from the work of Bernadette Bouchon-Meunier with her team: the calculation in fuzzy logic of reasoning and metaphorical schemes $[5,6]$.

\section{The Cognitive Psychology of Metaphorical Thinking}

There are psychological models of producing and of understanding metaphors. The model of Ortony [7] tries to apprehend the statements and the metaphors and proposes that the comparative statements are not necessarily true: their value of truth depends on the fact that the terms which they compare can be effectively assigned common predicates. Thus, the difference between literal comparisons and non-literal comparisons would arise from the fact that the terms linked in the literal comparisons have one or more significant predicates in common ((e.g., John is an Irish man, comparing Jogn to Irish people)). In non-literal comparisons, the common predicate, if a salient one, is only marginal for the topic (e.g., John is a fox, comparing John to foxes). Thus, nonliteral statements induce graduality. Ortony is also following Tversky by taking into account the features of both topic and vehicle and explains that a "feature is as an attribute or a predicate in a rather general sense: feature $X$ is something that we know about X"» and that according to Tversky the degree of similarity between two objects is a weighting function of the common features according to the features which distinguish them. Ortony argued that the essence of metaphoricity is salience imbalance; an imbalance that can be enhanced by attribute inequality. His proposals predict "that nonliteral similarity statements will tend to be much less reversible than literal similarity statements and that in cases in which reversals still result in meaningful comparisons, the meaning change will be greater for similes than for literals". 
Thus, Ortony attempts to explain the way in which people understand metaphors by proposing "the model of the salient imbalance". This model focuses on the fact that we must assign properties to the terms constituting the statements in order to be able to understand and interpret the metaphors. According to Ortony, there are a large number of properties that fight against each other (there is a confrontation of properties) and it is the most salient property that will allow to have a representation of a given object. Glucksberg and Keysar [8] offer their concept of understanding metaphor, which is based on a process of categorization. The authors believe that when an individual reads a metaphor, he activates the vehicle category (i.e. the term that gives metaphorical meaning) which allows him to have a representation of the situation. For example, the statement "Sophie is an ice cube" is a metaphor made up of a vehicle (i.e. ice cube) which qualifies a topic (i.e. Sophie). To understand the latter, the individual activates the category to which the ice cube vehicle belongs (i.e. cold things) before assigning the properties of the ice cube to the Sophie topical. Through their studies on the treatment of metaphorical statements, [7, 8] highlight the attribution of properties to objects via the transfer of knowledge. These studies show, moreover, that regardless of the differences in strategies implemented to process the statements, it is the transfer of knowledge which is at the origin of the recovery of information initiating the processing strategy.

Counterfactual reasoning, which is conventionally used in psychology as a diagnostic tool for evaluating creative potential, can help stem the phenomenon of metaphorical thought. According to [9-11] counterfactual reasoning makes it possible to transfer properties from one object to another object by an alternative attribution of categorized properties. This type of reasoning based on an alternative reality [12] implemented in our mind [1] allows a specific transfer of property between objects allowing not to refer to the prototype of the object to be considered but to what the object is not and could have been. For example, someone saying on Monday a counterfactual such as "If Paris was London" might imagine Parisian Museums being open on Mondays.

\section{The Fuzzy Logic Computation of Metaphors}

Since Zadeh's pioneering work on modifiers and on the one of Bouchon-Meunier and Colleagues [13, 14] which exploit the distribution of defined categories in a single universe, the mathematical transformation can be applied to different kinds of features, the concept of fuzzy relations being a generalization of the concept of relation, corresponding again to a fuzzy subset. In contrast to the preceding case concerning modifiers, this fuzzy set will not be constructed from a fuzzy set but from a value for two parts: the relation part of a specific feature and a category part. It is then possible to define mechanisms such that from a given feature and category, the fuzzy set representing the utterance can be constructed.

A major advance made by Bernadette Bouchon-Meunier that can be used for processing metaphor is the use of the main principle of fuzzy logic: that is to say the graduality of the membership function. Thus, the analogy as a metaphor (Juliet is the sun of Romeo) are fuzzy sets of particular types: analogy being calculated as a 
particular case of metaphor, with relations of transitivity, asymmetry and irreflexivity. This is due to her insight that the main principle of fuzzy logic, i.e. the graduality of membership function, might be a central core of metaphorical thinking and reasoning. There is a graduality of membership function that can be used both for "John is a man" and "John is a cat"; certainty (John is a man) being a special case of uncertainty (John is a cat). So, as crisp sets, that are representing precise and certain descriptions of objects, are to be regarded as particular cases of fuzzy sets [10], tautology as identity (the sun is the sun), analogy as comparison (the nuclear of the atom is as the sun of the solar system) and antilogy as metaphorical contradiction (Juliet is the sun although Juliet is not the sun) are all fuzzy sets of special kinds: identity being computed as a particular case of analogy, analogy being computed as a particular case of metaphor, with transitivity, asymmetry and irreflexivity relations [2].

As for analogy, concerning metaphorical reasoning, there are inter-domain relationships: a target object [T], that is to say the subject/tenor, is studied from the point of view of a source which is used as a vehicle (V) for the transmission of meanings [2]. Having comparisons between the same category (tautology), between different categories in the same domain (analogy), between different categories between domains or between inter-domain categories (metaphor), $\mathrm{T}$ and $\mathrm{V}$ can be calculated according to the evaluation of their "Proximity" through fuzzy modifiers to measure their similarity [6]. Thus, the proximity of two objects (i.e. the moon and the sun) as semantic distance (very close, close, far, very far) can be described through fuzzy sets which can manage the approximation. Indeed, the approximation of the proximity depends on the contextual role of $\mathrm{T}$ and $\mathrm{V}$ (the moon-sun proximity in "the moon is a sun" having a different value in "the sun is a moon"), according to the context (the moon proximity: sun in "tonight the moon is a sun" (is very bright in the night) being of a different value in "today at noon the moon is a sun") being very large; depending on the motif (the moon-sun proximity in "in your drawing; the moon is a sun "being of another value" in the sky, the moon is a sun”).

In the past, most of the cognitive models of metaphor understanding have adopted the approach according to which metaphor is an implicit comparison: understanding a metaphor " $\mathrm{X}$ (topic) is $\mathrm{Y}$ (vehicle)" consists in converting it into a simile " $\mathrm{X}$ (the topic) is like $Y$ (the vehicle)". This comparison-based model of metaphor understanding is a mechanism of property matching. This is why these models are confronted with the problem of measuring the similarity of properties as well as with the problem of calculating the distance between properties, which makes a simile literal or unliteral metaphoric.

As a difference with analogies, metaphors appear to be assertions that have a not reversible structure: if New York is a big apple, a big apple is not New York. Metaphor is inter-domains and the source is not a particular thing, but a category of things that serve to make the Target inherit the properties of the category. If the understanding of metaphor is based on the similarity computation of $\mathrm{T}$ and $\mathrm{V}$; the comparison would be symmetrical and reversible: $\mathrm{T}$ can be compared to $\mathrm{V}$ and $\mathrm{V}$ can be compared to $\mathrm{T}$. When two objects, situations or domains are comparable, either one or the other can serve as a source or as target. However, Metaphor is a particular case of comparison. Most of source-target comparison are oriented: a target can imply a source, the reverse being not true. 
There is a clear distinction between the objects of the physical world and the categories that humans use to represent them, in order to think, speak and communicate about them. Categories as sources of understanding can be used literally as in deduction and induction, but also in vague terms in metaphors. Like, we recommend that in both cases, the categorization of a target as being of a source type provides invisible features. As semantic relationships, metaphors are based on categorization. They activate a category and the functions attached to it. They also activate the superordinate categories and the functionalities attached to them.

For metaphors understanding [5] in which a source (for example, the sun) is a vehicle for transferring meanings to the target subject (for example, Juliet), the literature is about the notion that vehicles in metaphors are attributive categories. This categorization-based approach is mainly the Glucksberg's class inclusion model: a metaphorical statement of the type " $X$ is $Y$ ' is solved by searching for the category, (represented by the term $Y$ ), that provides source properties potentially relevant to the topic target $\mathrm{X}$. The general assumption underlying Glucksberg's approach is that understanding the metaphor involves including the topic in the source vehicle category and assigning it properties in that category that are consistent with what is known about the topic.

Following Glucksberg, we assume that the interpretation is constructed online and that knowledge of the subject comes into play at an early stage of processing by constraining the selection of functionalities. Bouchon-Meunier [15] noted that crisp sets representing precise descriptions and certain objects could be considered as special cases of fuzzy sets. Likewise, we argue that deduction, induction, abduction, induction, analogy and verbal metaphor are a special case of metaphorical thinking, from the least fuzzy-certain to the most fuzzy-uncertain; analogy being a special case of metaphor.

Bouchon-Meunier [11] proposed fuzzy reasoning based on prototypes for the fabrication and resolution of analogies. A fuzzy prototype of a category makes it possible to generate the typicity and the set of relevant objects and can therefore be used to match the source to the target; as Tversky suggests, the degree of typicity depends both on the resemblance to other objects of the same category and on the dissimilarity with objects of other categories. Thus, the metaphorical question that arises is "does the target gradually satisfy the prototype of the source category?" These are solutions for different modes of reasoning, including analogy and metaphor. Bouchon-Meunier and Valverde [12] were also tackling the problem of representing the similarities involved in metaphorical reasoning and used fuzzy relations to compare situations. Because vagueness involves various forms of reasoning, from real literal sentences to false non-literal sentences, it is a powerful method of calculation for human thinking and reasoning which is primarily reasoning based on metaphor and analogy. In addition, analogical and metaphorical sentences often include modifiers that Bouchon-Meunier and Marsala put at the core of fuzzy systems that can be interpreted. 


\section{The Fuzzy Logic Computation of Metaphors}

In short, according to Bouchon-Meunier [12], we use fuzzy relations to compare situations that can be used to model natural analogy: resemblance relations can be used to define a kind of metaphorical scheme compatible with approximate reasoning in fuzzy logic, with measures of satisfiability, likeness and inclusion. These fuzzy relationships can be seen as measures of a categorization process devoted to analogy and metaphor in order to convey knowledge from source to target.

Analogies and metaphors are common modes of thinking and reasoning, although based on a false categorization: "this lawyer is really a shark". So there is a powerful use of approximation and imprecision by the brain using analogies and metaphors through fuzzy inference. According to Bouchon-Meunier [11], progressive reasoning can be obtained using linguistic modifiers, the link between gradual reasoning and metaphorical reasoning corresponding to the use of a relation between the variations of $\mathrm{X}$ and the variations of $\mathrm{Y}$ expressed in progressive knowledge to deduce a value of $\mathrm{Y}$ from a given value of $\mathrm{X}$. Thus Bouchon-Meunier and her collaborators introduced a general framework representing the analogy, on the basis of a link between variables and measures of comparison between values of variables. This metaphorical scheme is a common description of several forms of reasoning used in fuzzy control or in the management of knowledge-based systems, such as deductive reasoning, inductive reasoning or prototypical reasoning, progressive reasoning.

\section{References}

1. Lakoff, G., Johnson, M.: Metaphors We Live By. University of Chicago Press, Chicago (2008)

2. Glucksberg, S.: The psycholinguistics of metaphor. Trends Cogn. Sci. 7(2), 92-96 (2003)

3. Tijus, C.: Analogy. In: A Fuzzy Dictionary of Fuzzy Modelling. Common Concepts and Perspectives. Tribute to Bernadette Bouchon-Meunier (in press)

4. Tversky, A.: Features of similarity. Psychol. Rev. 84, 327-352 (1977)

5. Bouchon-Meunier, B., Ramdani, M., Valverde, L.: Fuzzy logic, inductive and analogical reasoning. In: Ralescu, A.L. (ed.) FLAI 1993. LNCS, vol. 847, pp. 38-50. Springer, Heidelberg (1994). https://doi.org/10.1007/3-540-58409-9_4

6. Bouchon-Meunier, B., Marsala, C.: Fuzzy modifiers at the core of interpretable fuzzy systems. In: Tamir, D.E., Rishe, N.D., Kandel, A. (eds.) Fifty Years of Fuzzy Logic and its Applications. SFSC, vol. 326, pp. 51-63. Springer, Cham (2015). https://doi.org/10.1007/ 978-3-319-19683-1_4

7. Ortony, A.: Beyond literal similarity. Psychol. Rev. 86(3), 161-180 (1979)

8. Glucksberg, S.: Keysar, B: Understanding metaphorical comparisons: beyond similarity. Psychol. Rev. 97(1), 3-18 (1990)

9. Tijus, C., Barcenilla, J., Rougeaux, M., Jouen, F.: Open innovation and prospective ergonomics for smart clothes. In: 2nd International Conference on Ergonomics in Design (2014)

10. Candau, C., Ventalon, G., Barcenilla, J., Tijus, C.: Analogy and metaphors in images. In: Laurent, A., Strauss, O., Bouchon-Meunier, B., Yager, R.R. (eds.) IPMU 2014. CCIS, vol. 443, pp. 135-142. Springer, Cham (2014). https://doi.org/10.1007/978-3-319-08855-6_14 
11. Vivian, R., Brangier, E., Bornet, C.: How design the future hydrogen users' needs? A contribution of prospective ergonomics. In: Rebelo, F., Soares, M. (eds.) AHFE 2017. AISC, vol. 588, pp. 400-410. Springer, Cham (2018). https://doi.org/10.1007/978-3-31960582-1_40

12. Tijus, C.: Contextual categorization and cognitive phenomena. In: Akman, V., Bouquet, P., Thomason, R., Young, R. (eds.) CONTEXT 2001. LNCS (LNAI), vol. 2116, pp. 316-329. Springer, Heidelberg (2001). https://doi.org/10.1007/3-540-44607-9_24

13. Bouchon-Meunier, B., Yao, J.: Linguistic modifiers and gradual membership to a category. Int. J. Intell. Syst. 7, 25-36 (1992)

14. Brouard, C., Bouchon-Meunier, B., Tijus, C.: Modelling action in verbal command context with fuzzy subsets and semantic networks. In: Twentieth Annual Meeting of the Cognitive Science Society, pp. 179-184 (1998)

15. Bouchon-Meunier, B., Rifqi, M., Bothorel, S.: Towards general measures of comparison of objects. Fuzzy Set. Syst. 84(2), 143-153 (1996). Author, F.: Article title. Journal 2(5), 99-110 (2016) 